\title{
Treatment Patterns for Otitis Externa
}

\author{
Michael T. Halpern, MD, PbD, Cynthia S. Palmer, MSc, and Mindell Seidlin, MD
}

Background: Although otitis externa is a common and painful infection of the outer ear canal, there is little specific information available regarding current treatment patterns in the United States. We wanted to examine treatment patterns for otitis externa.

Methods: Data were analyzed from the 1993 National Ambulatory Medical Care Survey (NAMCS) and the 1993 National Hospital Ambulatory Medical Care Survey (NHAMCS) for adults and children treated for otitis externa. Data analyses included the reasons for physician visits, concomitant diagnoses, types of physicians seen, sources of payment, medical procedures administered, drugs prescribed, and patient disposition following a physician visit.

Results: Study results suggested that treatment patterns differ substantially for adults and children, as well as by physician specialty. Although otitis externa is frequently painful, few cases are classified as severe, and the data indicated that less than 20 percent of patients have concomitant diagnoses treatable by medication. Nevertheless, 40 percent of patients received both topical and systemic medication, and many of the oral antibiotics prescribed are not active against Staphylococcus aureus or Pseudomonas aeruginosa, the most common bacterial pathogens in otitis externa.

Conclusions: Appropriate treatment of localized otitis externa with topical antibiotics should eliminate the need for systemic medications. Addition of systemic medications can unnecessarily increase treatment costs and the likelihood of side effects, and could reduce the likelihood of patient compliance. ( $\mathrm{J}$ Am Board Fam Pract 1999;12:1-7.)

Acute otitis externa is a common, painful infection of the outer ear canal. The most common clinical manifestation of otitis externa is pain, followed by erythema, edema, itching, discharge, and hearing loss. ${ }^{1-4}$ Assessment of severity of otitis externa, rated by physicians on a scale from 0 to 3 based on physical findings and symptoms, indicates 44 percent of patients have mild disease, 43 percent have moderate disease, and 13 percent have severe dis-

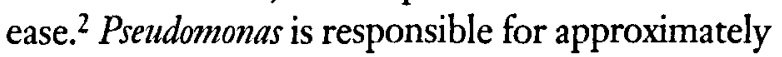
60 percent of infections, Staphylococcus for 15 percent, fungi for 10 percent, and other organisms for the remaining 15 percent of infections. ${ }^{5,6}$ Complications of otitis externa include ear canal stenosis, myringitis and tympanic membrane perforation, regional dissemination of infection (auricular cellulitis, chondritis, and parotitis), and progression

Submitted, revised, 19 May 1998.

From MEDTAP International (MTH, CSP), Bethesda, $\mathrm{Md}$, and Daiichi Pharmaceutical Corporation (MS), Ft. Lee, NJ. Address reprint requests to Michael T. Halpern, $M D$, PhD, MEDTAP International, 1701 Wisconsin Ave, Suite 600, Bethesda, MD 20814.

This study was funded by Daiichi Pharmaceutical Corporation, Ft. Lee, $\mathrm{NJ}$. to malignant otitis externa, which can be fatal. ${ }^{7}$

Treatment for otitis externa can include cleaning the ear as well as topical creams, ointments, or drops containing antiseptics, antibiotics, and steroids. ${ }^{8}$ There is, however, little specific information available regarding current treatment patterns in the United States The types of physicians treating otitis externa, procedures performed on otitis externa patients, and disposition of patients subsequent to treatment have not been examined. Thus, we undertook this study to evaluate otitis externa treatment patterns using US government national databases.

\section{Methods}

To evaluate treatment patterns, we analyzed data on otitis externa from two public databases: the 1993 National Ambulatory Medical Care Survey (NAMCS) ${ }^{9}$ and the 1993 National Hospital Ambulatory Medical Care Survey (NHAMCS). ${ }^{10}$ The NAMCS and NHAMCS are national probability sample surveys conducted by the Division of Health Care Statistics, National Center for Health Statistics, Centers for Disease Control and Prevention. These data are weighted to produce na- 
tional estimates that describe the utilization of ambulatory medical care services in the United States. Data sets from 1993 were the most recent available for this study.

The NAMCS is a survey of office-based physician practices evaluating patient office visits. Visits with federally employed physicians, visits occurring outside an office setting (eg, house calls, nursing home visits), and visits with anesthesiologists, pathologists, or radiologists were excluded. In 1993 , a total of 35,978 patient visits were evaluated from 1802 physicians.

The NHAMCS evaluates ambulatory care provided in hospital emergency and outpatient departments in the United States. For this study, only information on outpatient department visits was included. Outpatient visit data were collected at short-stay and general nonfederal hospitals. In $1993,28,357$ patient visits at 228 outpatient departments were included in the NHAMCS.

The probability sample design of the NAMCS and the NHAMCS allowed the sample data to be weighted to produce national estimates for the United States. Unweighted data are not used for analysis, as unweighted data ignore the disproportionate sampling used in the NHAMCS. Statistics from the NAMCS and the NHAMCS were derived by a multistage estimation procedure that produces essentially unbiased national estimates. The NAMCS and NHAMCS statistics reflect data concerning only a sample of patient visits, not a complete count of all the visits that occurred in the United States. Data presented in the NHAMCS and NAMCS include patient demographics (age, sex, race, ethnicity), provider characteristics (specialty, geographic region, metropolitan or nonmetropolitan status), expected source of payment; patient-supplied reason for visit, physician's diagnosis, patient disposition after the visit, and other medical care resource utilization (tests, procedures, or medications) associated with the visit.

For this study, we selected all patients with medical care visits having the ICD-9 ${ }^{11}$ diagnosis code 380.1 (infective otitis externa). Using the combined data from the NAMCS and NHAMCS, we found 190 outpatient episodes of otitis externa, corresponding to a weighted value of approximately 3.3 million outpatient visits. Results described here are based on weighted national data. Information regarding treatment patterns for pediatric patients (age 17 years or younger) are pre-
Table 1. Demographic Characteristics of Patient Visits.

\begin{tabular}{|c|c|c|c|c|}
\hline Visit & $\begin{array}{c}\text { Visit } \\
\text { Number }\end{array}$ & $(\%)$ & $\begin{array}{l}\text { Patient Age } \\
\text { Mean Years }\end{array}$ & $\begin{array}{l}\text { Percent } \\
\text { Female } \\
\text { Patients }\end{array}$ \\
\hline All & $3,314,423$ & $(100)$ & 31.0 & 52.1 \\
\hline Pediatric & $1,452,925$ & (43.8) & 9.3 & 52.2 \\
\hline Adult & $1,861,498$ & $(56.2)$ & 48.0 & 52.0 \\
\hline
\end{tabular}

sented separately from those for adults (age 18 years and older). As no identifying information is available in these data sets, longitudinal treatment patterns (ie, repeat visits or treatments by the same patients or relapse of otitis externa infections) and treatment outcomes could not be determined.

\section{Results}

Of the derived 3.3 million outpatient visits associated with otitis externa, 44 percent were pediatric visits (Table 1). The average ages of patients were 9 years for children and 48 years for adults. Just more than one half of both adults and children making visits were female. Among adults, most episodes of otitis externa were treated by family practice or general practice physicians $(55$ percent), otolaryngologists or head and neck surgeons (22 percent), and internal medicine physicians (12 percent). Pediatric patients received most care from pediatricians ( 57 percent), family practice or general practice physicians (26 percent), internists ( 7 percent), and otolaryngologists or head and neck surgeons ( 7 percent). The mean duration of physician visits was 12.5 minutes (12.2 minutes for children, 12.7 minutes for adults).

Table 2 displays the reasons for the otitis externa physician visits as reported by patients. $\mathrm{Pa}$ tients could list up to three reasons per visit. Earache was cited by a majority of both adult and child patients. Among adults, ear itch and other ear symptoms, discharge from the ear, and a plugged feeling in the ear were also cited. Approximately 5 percent of adults also visited the physician to obtain a prescription refill or for evaluation of a neck mass (presumably lymphadenopathy). Pediatric patients cited discharge from the ear and a plugged feeling in the ear at a rate similar to that of adults, but unlike adults, ear itch was cited as a reason by less than 1 percent of children. Pediatric patients described a number of systemic symptoms more frequently than did adults, including headache, nasal congestion, and cough. Adults 
Table 2. Reason for Physician Visit (Percent) for Otitis Externa.

\begin{tabular}{lrr}
\hline Symptom & Adult & Child \\
\hline Ear symptoms & & \\
Earache & 66.8 & 79.9 \\
Discharge from ear & 9.1 & 8.2 \\
Plugged feeling in ear & 5.9 & 5.9 \\
Trouble hearing & 2.2 & 3.5 \\
Ear itch and other symptoms & 15.5 & 0.6 \\
Dizziness & 1.5 & 0.0 \\
Otber symptoms & & \\
Headache & 0.2 & 4.8 \\
Nasal congestion & 0.7 & 6.1 \\
Chest congestion & 1.6 & 0.0 \\
Fever & 1.7 & 1.1 \\
Sore throat & 3.6 & 2.3 \\
Cough & 0.4 & 4.9 \\
Refill prescription & 4.8 & 0.0 \\
Neck mass & 5.7 & 0.0 \\
\hline
\end{tabular}

and children had similar rates of repeat visits for otitis externa (based on patients responding that they had previously seen this physician for the same condition).

Among adults, more than one half of patients had a follow-up appointment scheduled, whereas approximately one third were instructed to return if they felt it necessary (Table 3). Seven percent of adults had no scheduled follow-up and only a few had follow-up by telephone or with another physician. For children, more than one third had no scheduled follow-up. Slightly less than one third had a scheduled follow-up appointment, and one fourth were instructed to return as needed.

Up to eight different medical procedures can be listed in the NAMCS and NHAMCS for each outpatient visit. Few patients, however, received medical procedures during their physician visit for
Table 3. Patient Disposition (Percent) After Physician Visit for Otitis Externa.

\begin{tabular}{lrr}
\hline Disposition & Adult & Child \\
\hline No follow-up & 7.1 & 36.1 \\
Return as needed & 33.9 & 24.7 \\
Specified appointment & 53.7 & 31.0 \\
Telephone follow-up & 1.9 & 0.1 \\
Refer to other physician & 3.4 & 8.1 \\
\hline
\end{tabular}

otitis externa. The most common procedure for adults was ear irrigation ( 7 percent), with ear culture (6 percent) and audiometry ( 1 percent) being performed less frequently. Few children experienced ear irrigation ( 2 percent) or audiometry $(<1$ percent); no ear cultures were reported.

Table 4 displays concomitant diagnoses made at the outpatient visit for otitis externa. In the NAMCS and NHAMCS data sets, up to three diagnoses can be listed for each visit. Among adults, impacted cerumen was the most frequent concomitant diagnosis; this diagnosis can clearly be related to otitis externa. The second most prevalent concomitant diagnosis, however, was otitis media. Among children, otitis media was the most common concomitant diagnosis, reported at more than 22 percent of visits. Other concomitant diagnoses are less common and involve hearing loss or respiratory tract inflammation (pharyngitis, sinusitis, or bronchitis).

In the NAMCS and NHAMCS data sets, up to five medications (prescription and nonprescription) can be listed for each visit. For adult patients, no medications were prescribed at almost 20 percent of visits (Table 5). Topical (otic) medications were prescribed at more than 55 percent of visits and systemic medications at 65 percent; at more than 40 percent of visits, patients received pre-

Table 4. Concomitant Diagnoses Made at the Physician Visit for Otitis Externa.

\begin{tabular}{lcccc}
\hline & & \multicolumn{2}{c}{ Percent of Patients with Concomitant Diagnosis } \\
\cline { 3 - 5 } Concomitant Diagnosis & ICD-9* Code & All Patients & Adults & Children \\
\hline Impacted cerumen & 380.4 & 7.9 & 9.4 & 4.8 \\
Otitis media, unspecified & 382.9 & 12.1 & 7.0 & 22.6 \\
Sensorineural hearing loss & 389.1 & 1.1 & 1.6 & 0.0 \\
Acute pharyngitis & 462 & 2.1 & 1.6 & 3.2 \\
Chronic sinusitis, unspecified & 473.9 & 1.1 & 1.6 & 0.0 \\
Bronchitis, not otherwise specified & 490 & 1.6 & 1.6 & 1.6 \\
\hline
\end{tabular}

"Ninth Revision, International Classification of Diseases." 
Table 5. Type of Medication (Percent) Prescribed at Physician Visit for Otitis Externa.

\begin{tabular}{lccc}
\hline Medication & All Patients & Adults & Children \\
\hline None & 14.9 & 19.3 & 9.4 \\
Topical & 70.5 & 57.6 & 86.9 \\
Systemic & 54.4 & 65.4 & 40.2 \\
Both topical and systemic & 39.8 & 42.3 & 36.5 \\
\hline
\end{tabular}

scriptions for both topical and systemic medications. Pediatric patients showed similar treatment patterns, with more than 36 percent of visits resulting in prescriptions for both topical and systemic medications. Fewer children than adults, however, received no medications (9.4 percent versus 19.3 percent); furthermore, twice as many children received topical as systemic medications, whereas the proportions among adults were nearly equal.

Tables 6 and 7 provide details regarding the medications prescribed overall and by physician specialty. As shown at the end of the total column for these tables, the mean number of medications prescribed was 1.4 for children and 2.0 for adults. Most pediatric patients received a ophthalmic suspension of neomycin, polymyxin $B$, and hydrocortisone (Cortisporin) (Table 6). In addition, more than 13 percent of pediatric patients received generic versions of the neomycin-polymyxin B- hydrocortisone combination (Otocent, Pediotic, or Coly-Mycin). A greater number of pediatric patients received anesthetic ear drops than did adults. The results listed by physician specialty are less certain than the totals, because of the few visits represented. Most visits with family physicians, general practitioners, or pediatricians and more than 25 percent of visits with otolaryngologists resulted in prescriptions for the neomycin-polymyxin B-hydrocortisone suspension; in contrast, internal medicine physicians prescribed a generic medication (Otocent) to more than 40 percent of patients. Almost 60 percent of children seen by internal medicine physicians received anesthetic ear drops.

Among adult patients, the neomycin-polymyxin B-hydrocortisone suspension (otic or ophthalmic preparation) was the most common topical medication prescribed (Table 7). An acetic acid solution (Vosol) and ophthalmic prednisolone (Pred-G) were the next most commonly prescribed topical medications. All other topical medications were prescribed at lower frequencies. Most prescribed systemic medications were antibiotics. Analgesics or nonsteroidal anti-inflammatory drugs were prescribed at approximately 20 percent of adult physician visits. Seventeen percent of adult patients also received bronchodilators (albuterol or pirbuterol) or nasal corticosteroids (Vancenase). The purpose of the nasal corticosteroids might have been to

Table 6. Medications (Percent) Prescribed to Pediatric Patients by Physician Specialty.

\begin{tabular}{|c|c|c|c|c|c|}
\hline Medications & $\begin{array}{l}\text { Family, } \\
\text { eneral Practice }\end{array}$ & Pediatrics & $\begin{array}{c}\text { Internal } \\
\text { Medicine }\end{array}$ & Otolaryngology & Total \\
\hline \multicolumn{6}{|l|}{ Topical } \\
\hline Neomycin-polymyxin B-hydrocortisone (Cortisporin) & 56.5 & 70.1 & 0.0 & 21.1 & 56.9 \\
\hline Neomycin-polymyxin B-hydrocortisone (Pediotic) & 3.4 & 10.7 & 0.0 & 0.0 & 6.8 \\
\hline Hydrocortisone-acetic acid otic solution (Vosol HC) & 0.0 & 10.7 & 0.0 & 0.0 & 6.0 \\
\hline Neomycin-polymyxin B-hydrocortisone (Cortisporin) ophthalmic & 0.0 & 8.6 & 0.0 & 4.0 & 5.0 \\
\hline Anaesthetic ear drops & 0.0 & 0.0 & 58.2 & 6.1 & 4.4 \\
\hline Neomycin-hydrocortisone-thonzonium (Coly-Mycin) & 12.6 & 0.0 & 0.0 & 10.1 & 3.9 \\
\hline Hydrocortisone (Otocort) & 0.0 & 0.0 & 41.8 & 0.0 & 2.9 \\
\hline Pentamycin sulfate (Gentak) & 0.0 & 0.0 & 0.0 & 16.4 & 1.1 \\
\hline \multicolumn{6}{|l|}{ Systemic and other } \\
\hline Penicillin & 4.7 & 17.4 & 41.7 & 15.5 & 16.5 \\
\hline Analgesic & 4.7 & 8.6 & 58.2 & 0.0 & 10.1 \\
\hline Sulfonamide & 27.2 & 0.0 & 0.0 & 0.0 & 8.9 \\
\hline Macrolide & 12.6 & 1.3 & 58.2 & 0.0 & 8.0 \\
\hline Cephalosporin & 0.0 & 10.7 & 0.0 & 10.1 & 6.8 \\
\hline Nasal decongestant & 12.6 & 0.0 & 0.0 & 0.0 & 5.2 \\
\hline Mean number per patient & 1.3 & 1.4 & 2.6 & 0.9 & 1.4 \\
\hline
\end{tabular}

4 JABFP Jan.-Feb. 1999 Vol. 12 No. 1 
Table 7. Medications Prescribed to Adult Patients (Percent) by Physician Specialty.

\begin{tabular}{|c|c|c|c|c|}
\hline Medications & $\begin{array}{c}\text { Family, } \\
\text { General Practice }\end{array}$ & $\begin{array}{l}\text { Internal } \\
\text { Medicine }\end{array}$ & Otolaryngology & Total \\
\hline \multicolumn{5}{|l|}{ Topical } \\
\hline Neomycin-polymyxin B-hydrocortisone (Cortisporin) & 22.7 & 56.0 & 40.2 & 34.3 \\
\hline $\begin{array}{l}\text { Neomycin-polymyxin B-hydrocortisone (Cortisporin) } \\
\text { ophthalmic }\end{array}$ & 10.8 & 0.0 & 1.8 & 6.2 \\
\hline Acetic acid otic solution (Vosol) & 5.3 & 0.0 & 0.0 & 2.8 \\
\hline Hydrocortisone-acetic acid otic solution (Vosol HC) & 0.0 & 0.0 & 6.5 & 4.7 \\
\hline Prednisolone acetate (gentamycin sulfate) & 0.0 & 44.0 & 0.0 & 5.0 \\
\hline Aluminum acetate (Domeboro) & 0.0 & 0.0 & 9.6 & 2.0 \\
\hline Dexamethasone (NeoDecadron) ophthalmic & 0.0 & 0.0 & 1.5 & 0.3 \\
\hline Neomycin-hydrocortisone-thonzonium (Coly-Mycin) & 0.0 & 0.0 & 3.2 & 0.9 \\
\hline Prednisolone acetate - sulfacetamide-sodium (Vasocidin) & 0.0 & 0.0 & 1.0 & 0.3 \\
\hline Hydrocortisone (Clioquinol) & 0.0 & 0.0 & 1.3 & 0.3 \\
\hline Anaesthetic ear drops & 0.0 & 0.0 & 4.4 & 0.9 \\
\hline \multicolumn{5}{|l|}{ Systemic and other } \\
\hline Cephalosporin & 23.3 & 0.0 & 6.2 & 21.2 \\
\hline Macrolide & 17.6 & 35.5 & 6.2 & 16.0 \\
\hline Ciprofloxacin & 21.0 & 0.0 & 7.6 & 12.7 \\
\hline Nonsteroidal anti-inflammatory drug & 17.8 & 20.5 & 0.0 & 12.1 \\
\hline Beclomethasone (Vancenase) & 20.5 & 0.0 & 1.5 & 11.2 \\
\hline Analgesic & 14.6 & 0.0 & 0.0 & 8.2 \\
\hline Penicillin & 3.4 & 44.0 & 3.8 & 7.8 \\
\hline Nasal decongestant & 10.8 & 0.0 & 3.0 & 6.5 \\
\hline Bronchodilator & 10.8 & 0.0 & 0.0 & 6.2 \\
\hline Sulfonamide & 10.3 & 0.0 & 1.5 & 5.8 \\
\hline Antihistamine & 9.8 & 0.0 & 2.6 & 5.7 \\
\hline Mean number per patient & 2.3 & 2.2 & 1.3 & 2.0 \\
\hline
\end{tabular}

treat a secondary or concurrent eustachian tube dysfunction.

Approximately one third of adult and child visits were paid for through private insurance (nonmanaged care). A similar proportion of adult visits were paid for by Medicare. Approximately 17 percent of adult visits were insured by managed care or health maintenance organization (HMO), 19 percent were paid for out-of-pocket by the patient, and 7 percent of visits were paid for by Medicaid. Among children, nearly one half of all visits were insured by HMOs, 17 percent were associated with Medicaid insurance, and 5 percent were paid for out-of-pocket.

For adults and children combined, Table 8 summarizes the sources of payment by type of physician. Internal medicine physicians and otolaryngologist, head and neck surgeons received a larger percentage of their payments from private insurers than other sources. Pediatricians received almost 60 percent of payments from HMO-pre- paid sources. There were smaller differences across sources of payment for family physicians and general practitioners.

\section{Discussion}

Otitis externa is a common condition, and most cases are treated by family physicians and general practitioners. The main patient complaint associated with this condition is earache (corresponding to pain, the most common symptom reported in the literature). Other symptoms, such as discharge, trouble hearing, and itch, occur less frequently than reported in the literature.

The differences between adult and pediatric visit outcomes (Table 3) suggest that otitis externa is viewed as more serious among adults, requiring scheduled follow-up, whereas no follow-up was scheduled with 70 percent of pediatric visits. Adults also have a greater likelihood of being seen by an otolaryngologist, having a procedure performed, and receiving a greater number of pre- 
Table 8. Payment Source (Percent) by Physician Specialty.

\begin{tabular}{lccrr}
\hline Specialty & $\begin{array}{c}\text { Health Maintenance } \\
\text { Organization, Prepaid }\end{array}$ & $\begin{array}{c}\text { Other } \\
\text { Private Insurance }\end{array}$ & Government & Out-of-Pocket \\
\hline Family practice, general practice & 23.2 & 26.6 & 33.0 & 17.2 \\
Internal medicine & 29.8 & 46.1 & 0.0 & 24.1 \\
Otolaryngology, head and neck surgery & 9.7 & 41.8 & 31.0 & 17.5 \\
Pediatrics & 58.9 & 31.2 & 9.8 & 0.0 \\
\hline
\end{tabular}

scribed medications. Nevertheless, more than twice as many children were referred to other physicians (presumably specialists) as were adults. One possible explanation for this difference in referral patterns is that in cases where otitis externa is persistent or severe, physicians are more inclined to refer children than adults for specialist care. Also, more adults receive care from otolaryngologists than do children; these specialists might be unlikely to refer their patients to other physicians, contributing to the differential rate of referrals. There is probably a correlation between insurance coverage and referral pattern, as well as insurance coverage and type of medication prescribed.

Otitis media is the most frequent concomitant diagnosis reported from the otitis externa visit. In more than 22 percent of physician visits, children with otitis externa also have otitis media diagnosed. Physicians might diagnose both otitis conditions based on symptoms if the tympanic membrane cannot be visualized. Alternatively, generalist physicians might have difficulty distinguishing the two conditions, or might record both diagnoses to justify prescription of both topical and systemic medications.

A frequently recommended treatment for otitis externa is meticulous cleaning of the external auditory meatus when disease severity and symptoms (eg, pain) permit. While physicians can suction or swab ear canal debris as part of the cleaning procedure, only 7 percent of physician visits for adults and 2 percent of physician visits for children reported ear irrigation. The methods and impact of cleaning the auditory canal on disease outcome remain uncertain.

The most common therapy for otitis externa is a topical agent containing antibiotics (an aminoglycoside and polymyxin B) and a corticosteroid. These medications are thought to both reduce the inflammation and treat the (putative) underlying pathogen. These medications, however, require four administrations per day (based on package labeling), and as with other forms of medications, compliance decreases with increased number of daily administrations. Compliance might also be affected by stinging or burning, which commonly occurs following administration of otic medication. ${ }^{12}$ Furthermore, otic aminoglycoside therapy can lead to ototoxicity or cutaneous sensitization. ${ }^{13}$ It might be useful to evaluate compliance with these topical medications, including the impact of symptoms experienced in administering these medications on compliance.

Approximately 40 percent of patients had both systemic and topical medications prescribed, mainly antibiotics. Many of the oral antibiotics prescribed are not active against Stapbylococcus aureus or Pseudomonas aeruginosa, the most common bacterial pathogens in otitis externa. ${ }^{7,14}$ Appropriate treatment of localized otitis externa with topical antibiotics should remove any need for the additional of systemic medications, which can increase both costs and the likelihood of side effects, and potentially reduce patient compliance.

There has been only minimal examination regarding the costs of otitis externa. In a study by van Asperen et al, ${ }^{4}$ performed in the Netherlands, 19 percent of 98 otitis externa patients continued having symptoms after 4 weeks of treatment with prescribed ear drops. A physician was consulted twice by 37 percent of the patients and 3 or more times by 14 percent of patients. Thirty-five percent of patients received a prescription more than once, and 6 percent received a prescription more than twice. Discontinuation of daily activities was reported by 36 percent of patients, with a median duration of 4 days. Twenty-one percent of patients had to rest in bed for a median duration of 3 days.

The database analysis only included patients for whom otitis externa was the primary diagnosis. Treatment patterns might differ among patients for whom this condition is the second or third diagnosis for the visit. In addition, the data did not 
permit examination of longitudinal treatment patterns, failure or relapse rates, or total cost of illness. Even so, the treatment patterns evaluated in the database and published literature suggest that there might be considerable resource utilization and costs associated with the care for otitis externa. Beyond the direct medical costs, there are also indirect costs associated with missed work or school and decreased activity. These costs might be even greater than those for medical resource utilization. Prospective evaluation of treatment efficacy, including compliance, and the direct and indirect costs of otitis externa in the United Sates would be useful, and would permit evaluation of the cost-effectiveness of new treatments for otitis externa.

\section{References}

1. Calderon R, Mood EW. An epidemiological assessment of water quality and "swimmer's ear." Arch Environ Health 1982;37:300-5.

2. Cassisi N, Davidson T, Cohn A, Witten BR. Diffuse otitis externa: clinical and microbiologic findings in the course of a multicenter study on a new otic solution. Ann Otol Rhinol Laryngol 1986;39(Suppl):1-16.

3. Manni JJ, Kuylen K. Clinical and bacteriological studies in otitis externa in Dar es Salaam, Tanzania. Clin Otolaryngol 1984;9:351-4.

4. van Asperen IA, de Rover CM, Schijven JF, Oetomo SB, Schellekens JF, van Leeuwen NJ, et al. Risk of otitis externa after swimming in recreational fresh water lakes containing Pseudomonas aeruginosa. BMJ
1995;311:1407-10.

5. Farmer HS. A guide for the treatment of external otitis. Am Fam Physician 1980;21(6):96-101.

6. Russell JD, Donnelly M, McShane DP, Alun-Jones T, Walsh M. What causes acute otitis externa? J Laryngol Otol 1993;107:898-901.

7. Bojrab D, Bruderly T, Abdulrazzak Y. Otitis externa. Otolaryngol Clin North Am 1996;29:761-82.

8. Hirsch BE. Infections of the external ear. Am J Otolaryngol 1992:13:145-55.

9. Tenney JB, White KI, Williamson JW. National Ambulatory medical care survey: background and methodology, United States, 1967-1972; a report on feasibility studies of methods developed for collecting national ambulatory medical care data from practicing office-based physicians in the United States. Vital and health statistics, series 2, no 61. Rockville, Md: National Center for Health Statistics, 1974. (DHEW publication no (HRA) 74-1335.

10. National hospital ambulatory care survey. CD ROM series 13, No 10. SETS version 1.22a. Rockville, Md: National Center for Health Statistics, July 1997.

11. Ninth revision, international classification of diseases. In: Manual of the international statistical classification of diseases, injuries, and causes of death. Geneva: World Health Organization, 1977.

12. Slack RW. A study of three preparations in the treatment of otitis externa. J Laryngol Otol 1987;101: 533-5.

13. Physicians' genRx. Riverside, Conn: Denniston Publishers, 1995.

14. Cantor RM. Otitis externa and otitis media. A new look at old problems. Emerg Med Clin North Am $1995 ; 13(2): 445-55$. 